\title{
The initial process of enamel prism arrangement and its relation to the Hunter-Schreger bands in dog teeth
}

\author{
Yoshinori Hanaizumi ${ }^{1}$, Rumi Yokota ${ }^{2}$, Takanori Domon ${ }^{1}$, Minoru Wakita, ${ }^{1}$ and Yukisige \\ Kozawa
}

${ }^{1}$ Division of Oral Functional Science Graduate School of Dental Medicine Hokkaido University, Sapporo; and ${ }^{2}$ Department of Histology Cytology and Developmental Anatomy Nihon University School of Dentistry, Matsudo, Japan

Summary. The three-dimensional architecture of enamel prisms at early stages of enamel formation and its spatial relationship to the Hunter-Schreger bands were examined in canine tooth germs by light and electron microscopy. In serial semithin sections of demineralized tooth germs tangential to the enamel-dentin junction, a straight row of enamel prisms was depicted along the longitudinal tooth axis at the level of the enamel-dentin junction and then their three-dimensional arrangement was reconstructed using computer software. The spatial arrangement of the groups of enamel rods oriented in specific sideward directions was also reconstructed in deep layers of the enamel.

Initially, all enamel prisms were parallel to perpendicular toward the enamel-dentin junction, but at $10 \mu \mathrm{m}$ from the enamel-dentin junction, some small specks, or groups of enamel prisms - tilting to the right or the leftemerged as small islands. In each speck of enamel prism, the inclined prisms were uniformly oriented in a sideward direction and gradually expanded their boundary until merging with the neighboring specks inclined in the same direction. Consequently, at $50 \mu \mathrm{m}$ from the enamel-dentin junction, the group of enamel prisms oriented either to the right or the left formed alternately arranged horizontal

Received August 19, 2008; revised December 17, 2008

Address for correspondence: Dr. Yoshinori Hanaizumi, Division of Oral Functional Science, Hokkaido University Graduate School of Dental Medicine, Kita 13, Nishi 7, Kitaku, Sapporo 060-8586, Japan

Tel: + 81-11-584-0055, Fax: + 81-11-584-0055

E-mail: hanaizum@cocoa.ocn.ne.jp belt-like zones, corresponding to the parazone or the diazone of the Hunter-Schreger bands.

Reversed images of scanning electron-micrographs of the exposed surfaces of the developing enamel revealed round and bulb-like profiles of Tomes' processes at early amelogenesis and its changes into a characteristic structure combined with flat secretory and enclosing nonsecretory faces that dictated the orientation of corresponding enamel prisms.

The results suggest that the groups of enamel prisms oriented in sideward directions first appear as small island-like specks near the enamel-dentin junction, which later merge and form alternating horizontal belt-like zones as a consequence of morphological changes of the Tomes' processes. However, the mechanisms whereby the functional grouping of secretory ameloblasts with similarly oriented Tomes' processes is induced are yet to be determined.

\section{Introduction}

The Hunter-Schreger bands appear on the longitudinally cut surfaces of tooth enamel as characteristic alternating bands that correspond respectively to the zones of longitudinally and transversely cut prisms extending from the enamel-dentin junction to the enamel surface. The pattern of Hunter-Schreger bands is species and tooth-type specific, and, in various mammals, the threedimensional courses and arrangement of enamel prisms and their relation to the Hunter-Schreger bands have been extensively examined by a variety of methods (Gustafson, 1945; Erausquin, 1949; Kawai, 1951,1955; Mortell, 1956; 
Applebaum, 1960; Heuser, 1961; Boyde, 1964,1969; Lester, 1965; Sundstrom, 1966; Osborn, 1965, 1968a, b, c,1990; Kozawa, 1978, 1984; Skobe, 1980; Hirota, 1982; Koenigswald et al., 1987).

The spatial arrangement of enamel prisms is closely related to the morphology and direction of the Tomes' processes at the apical end of ameloblasts. Many investigations have examined the three-dimensional features and arrangement patterns of Tomes' processes (Skobe, 1976, 1977; Wakita et al., 1981), Tomes' process pits on the developing enamel surface (Boyde, 1964,1969; Warshawsky et al.,1981; Nishikawa, 1992), or resin replicas of Tomes' process pits corresponding to the structure of Tomes' processes (Kindaichi et al., 1981; Ohara et al., 1990) by scanning electron microscopy. These studies reported that the direction and arrangement of the secretory faces of Tomes' processes shifted periodically and clamed that ameloblasts could be characterised as those with Tomes' processes having secretory faces inclined in the same direction (Boyde, 1964, 1969; Kindaichi et al.,1981; Wakita et al.,1981). However, the microscopic images of Tomes' processes and their related structures in these studies were often unclear, most probably due to mechanical damage during preparation.

We previously, attempted a three-dimensional reconstruction of the enamel prism structures of a dog tooth by out of serial tangential sections of growing enamel using a computer simulation program and also examined the detailed features of growing enamel surfaces by scanning electron microscopy after the chemical removal of cellular elements by the cellmaceration method (Ohtani et al., 1988; Ushiki and Ide, 1990). Data showed that a group of ameloblasts with secretory faces of Tomes' processes similarly inclined formed a group of enamel prisms oriented in the same sideward direction, and that such a group of enamel prisms formed belt-like zones on tangentially cut surfaces, corresponding to the parazone or diazone of the HunterSchreger bands on the longitudinally cut tooth surfaces (Hanaizumi et al., 1996, 1998).

Although the arrangement of Tomes' processes has been thought to be related to the distribution of enamel prisms, as described above, there is still no precise knowledge of how the groups of similarly inclined prisms (i.e., the groups of ameloblasts having Tomes' processes with similarly oriented secretory surfaces) emerge at early enamel formation. In the present study, observations of three-dimensional features of Tomes' processes as well as the corresponding enamel prisms as a function of distance from the enamel-dentin junction serve to answer this fundamental question.

\section{Materials and Methods}

All animal experimentation followed the Guide for the Care and Use of Laboratory Animals, Hokkaido University Graduate School of Dental Medicine, which is based on the Guide for the Care and Use of Laboratory Animals (NIH 1985).

\section{Light microscopy}

A 1-month-old dog was anesthetized with an intravenous injection of Nembutal $(0.5 \mathrm{ml} / \mathrm{kg}$ body weight) and perfused with a mixture of a $0.1 \mathrm{M}$ phosphate buffered solution of $2 \%$ paraformaldehyde and $1.25 \%$ glutaraldehyde at $\mathrm{pH}$ 7.4. The jaws were removed and immersed in the same fixative overnight. Permanent tooth germs were dissected out, and canine and molar tooth germs were demineralized in 5\% EDTA $(\mathrm{pH} 7.4)$ for three weeks. The tooth germs were postfixed in $1 \%$ osmium tetroxide for $3 \mathrm{~h}$, dehydrated in ethanol, and embedded in Spurr's resin.

First, the canine tooth germs were cut in a direction parallel to the tooth axis up to the longitudinal plane (plane LA and LB in Fig.1) vertical to the enamel-dentin junction that was obtained; next, the block was remounted on the ultramicrotome at right angles to the original sectioning plane with the tooth axis unchanged, and serial

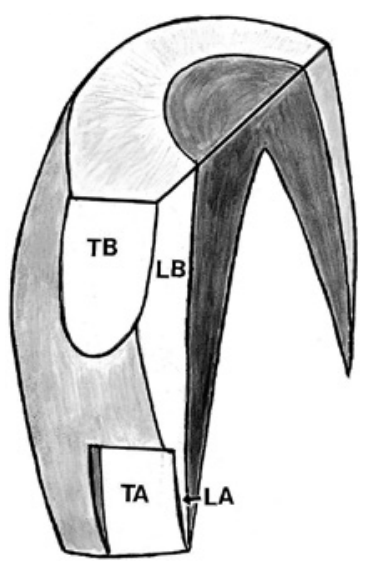

Fig.1. Diagram showing the direction of sectioning planes. LA: longitudinal plane in the cervical region parallel to the tooth axis, LB: longitudinal plane in the thick enamel region, TA: tangential plane in the cervical region parallel to the enamel-dentin junction, TB: tangential plane in the thick enamel region. 

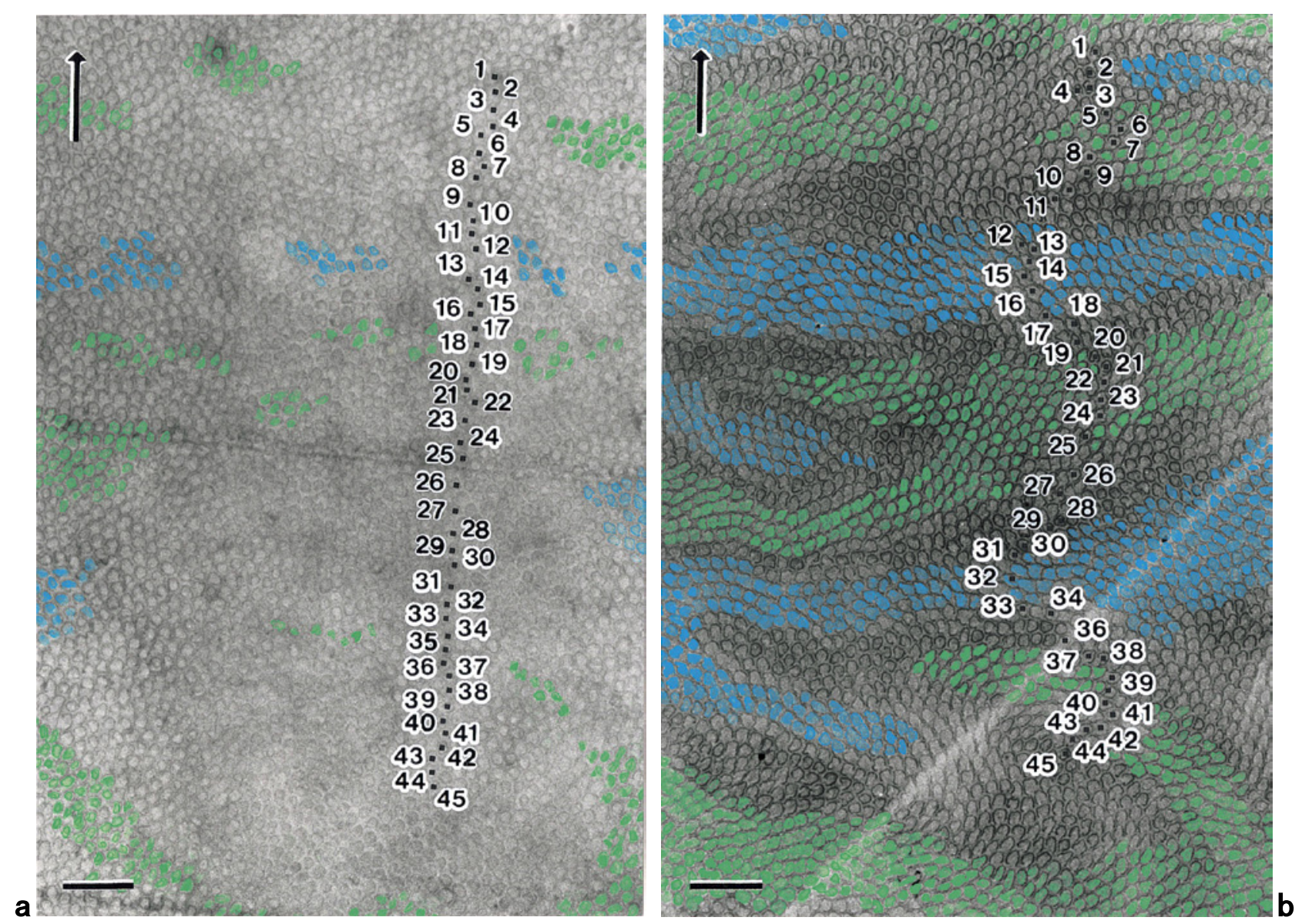

Fig. 2. Light micrographs of tangential semithin section $5 \mu \mathrm{m}$ (a) and $45 \mu \mathrm{m}$ (b) from the enamel-dentin junction. a: First section in a set of the sections used for reconstruction of the enamel prisms. Enamel prisms in a straight row in the occlusal direction (arrow) are selected and numbered 1-45 from the top. The direction of enamel prisms here could be estimated from the inclination of the top of the prism sheaths; blue prisms orient to the right, and green prisms orient to the left. b: Section $40 \mu \mathrm{m}$ from the plane of section a. The row of numbered enamel prisms forms a wavy sine-like curve. Blue and green prisms lie in belt-like zones roughly perpendicular to the occlusal direction (arrow). Scale bars: $30 \mu \mathrm{m}$

one $\mu \mathrm{m}$ thick tangential sections were cut parallel to the enamel surface in the direction toward the enamel-dentin junction at the primary cervical (plane TA in Fig.1) and the thick enamel region (plane TB in Fig.1). For the threedimensional reconstruction, the latter serial tangential semithin sections (plane TB in Fig.1) were used. All sections were stained with methylene blue and azure II, and photographed with a light microscope (Nikon Microphot FXA).

\section{Scanning electron microscopy}

Some of the molar tooth germs, dissected as described above, were immersed in a buffered $2 \% \mathrm{OsO}_{4}$ solution for several hours at $4{ }^{\circ} \mathrm{C}$. To remove the enamel organ and expose the enamel surface without causing mechanical damage, the remaining molar tooth germs were processed for the cell-maceration method (Ohtani et al., 1988; Ushiki and Ide,1990) to remove cellular elements and the basal laminae, leaving the extracellular matrix: The fixed specimens were immersed in a $5 \%$ aqueous solution of $\mathrm{NaOH}$ for 2-3 days at room temperature and rinsed with distilled water for 1-2 days, these processes being repeated several times. The $\mathrm{NaOH}$-treated specimens were then dehydrated, transferred to isoamyl acetate, and critical-point dried with liquid $\mathrm{CO}_{2}$. The dried specimens 

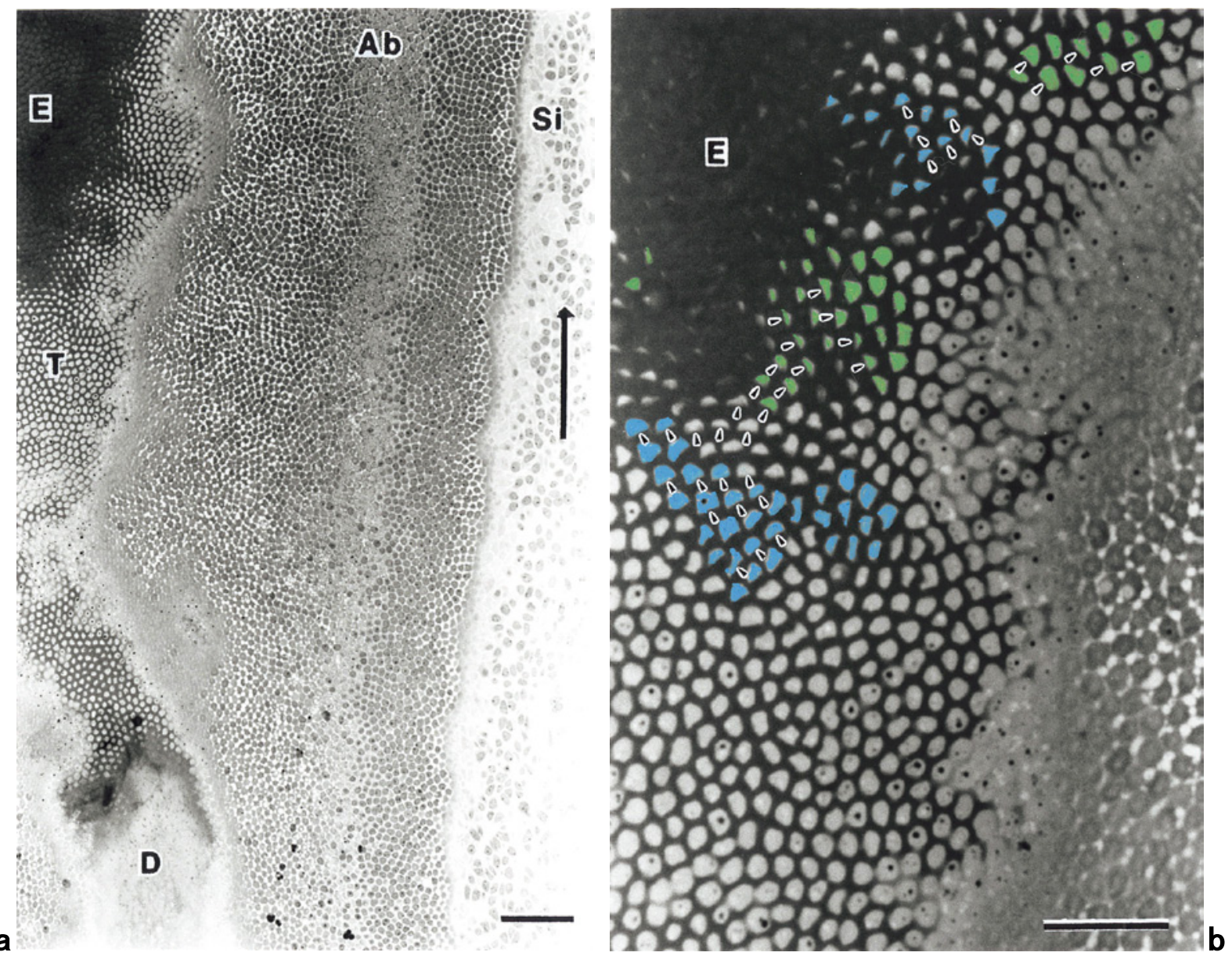

Fig. 3. Light micrographs from a tangential semithin section along the enamel-dentin junction at the site of initial enamel deposition in the cervical region (TA in Fig.1). a: Low magnification showing the areas of enamel (E), dentin (D), cross sections of ameloblasts $(\mathrm{Ab})$, and stratum intermedium ( $\mathrm{Si}$ ) together. The occlusal direction is indicated by the arrow. The cross-cut profiles of Tomes' processes (T) of ameloblasts at the site of initial enamel secretion are rounded and their arrangement is somewhat irregular. Scale bar: $50 \mu \mathrm{m}$. b: Enlargement of the upper later stage of initial enamel formation of a. The secretory faces of the Tomes' processes are twisted to the right or left (arrowheads). Some rough outlines of the specks of ameloblasts comprise those with similarly inclined Tomes' processes. Tomes' processes oriented to the right are colored blue, and Tomes' processes oriented to the left are colored green. Ab: ameloblast, T: Tomes' process, E: enamel. Scale bars: $30 \mu \mathrm{m}$

were then fixed on aluminum stubs with double-sided tape and coated with platinum in an ion coater (Hitachi E-1032) before observation under a scanning electron microscope (Hitachi S-4000) at an accelerating voltage of $10 \mathrm{kV}$.

\section{Three-dimensional reconstruction}

The three-dimensional reconstruction followed the method of Hanaizumi (1992) and Hanaizumi et al. (1994):
Light microscopic images of tangentially cut serial semithin sections were enlarged on photographic printing paper, and the boundaries between the groups of enamel prisms traced on tracing paper. Using the outlines of each section as a reference, the images of the enamel prisms and the boundaries were read serially into a personal computer (NEC PC-9801 VM) using software for threedimensional graphic analysis (Cosmozone 2SA Nikon). The reconstructed images were photographed from the computer display. 

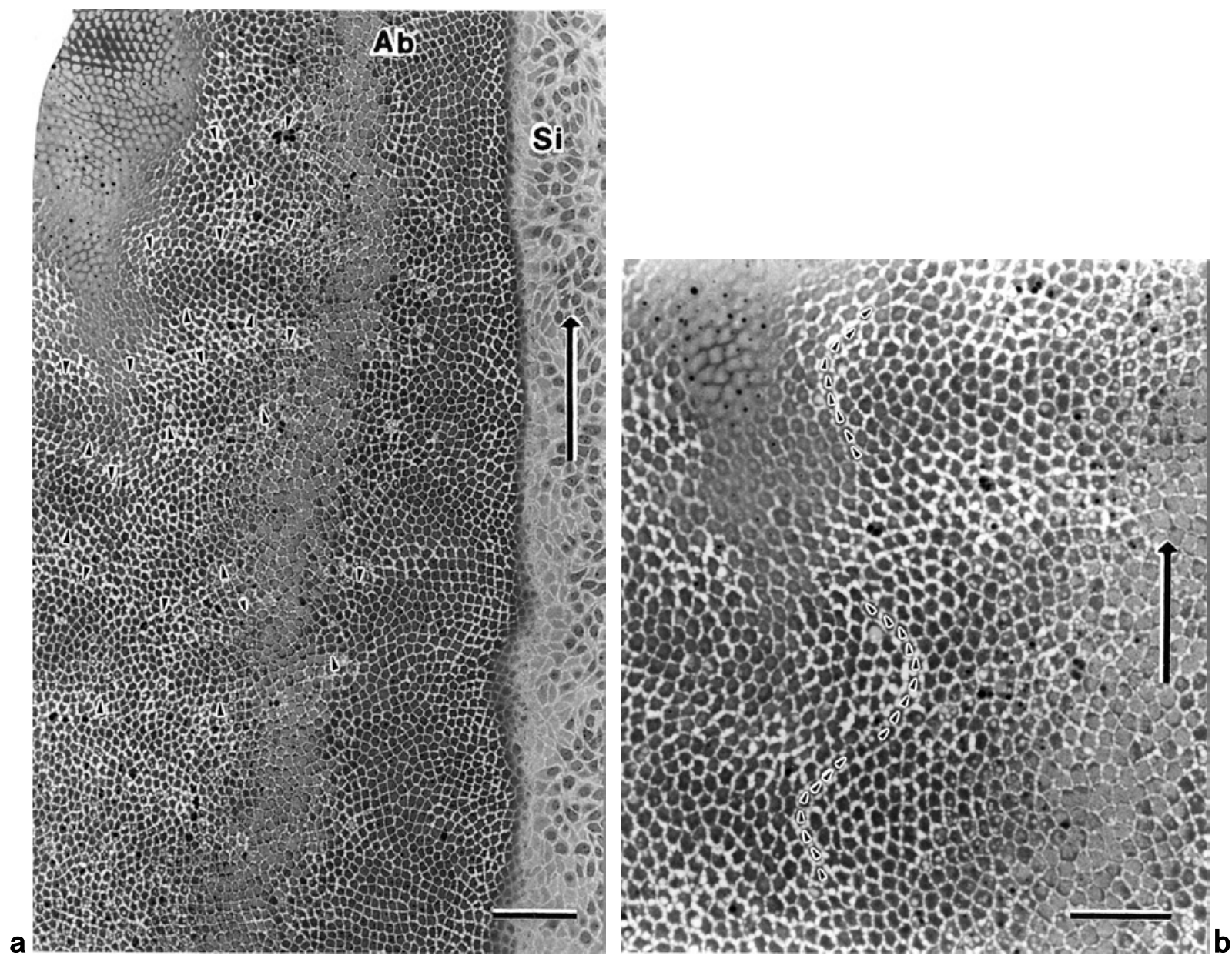

Fig. 4. Light micrographs of a tangential semithin section from the cervical regions more occlusal than in Figure 3. a: Cross sections of ameloblasts ( $\mathrm{Ab}$ ) and stratum intermedium ( $\mathrm{Si}$ ). The ameloblasts with densely stained cytoplasm are distributed in groups and discernible as belt-like bands (arrowheads). The outlines of the cell bodies of respective ameloblasts at the level of the distal terminal web exhibit a horizontally extending belt-like arrangement. The occlusal direction is indicated by the arrow. Scale bar: $50 \mu \mathrm{m}$. b: Enlargement of a. Rows of ameloblast extend in a wavy manner to form a sinusoidal curve (arrowheads) toward the occlusal direction (arrow). Ab: ameloblast, Si: stratum intermedium. Scale bar: $30 \mu \mathrm{m}$

\section{Results}

\section{Light microscopy}

A tangential semithin section $5 \mu \mathrm{m}$ distant from the enamel-dentin junction (plane TB in Fig.1) was used as the initial plane for reconstruction of the enamel prisms. A straight row of enamel prisms parallel to the occlusal direction was selected for the reconstruction and numbered serially from the top (Fig.2a).
The enamel prism sheath represents a fossilized path traced by the Tomes' processes during enamel secretion (Boyde,1967), and the secretory face of Tomes' process is at right angles to the longitudinal axis of prisms (Boyde, 1967,1969; Wakita et al., 1981; Hanaizumi et al., 1994). Considering this, the direction of enamel prisms here was estimated from the inclination of the top of the crosscut images of prisms sheaths; and the prisms oriented to the right were colored blue, and prisms oriented to the left were colored green (Fig. 2a). The marked enamel 


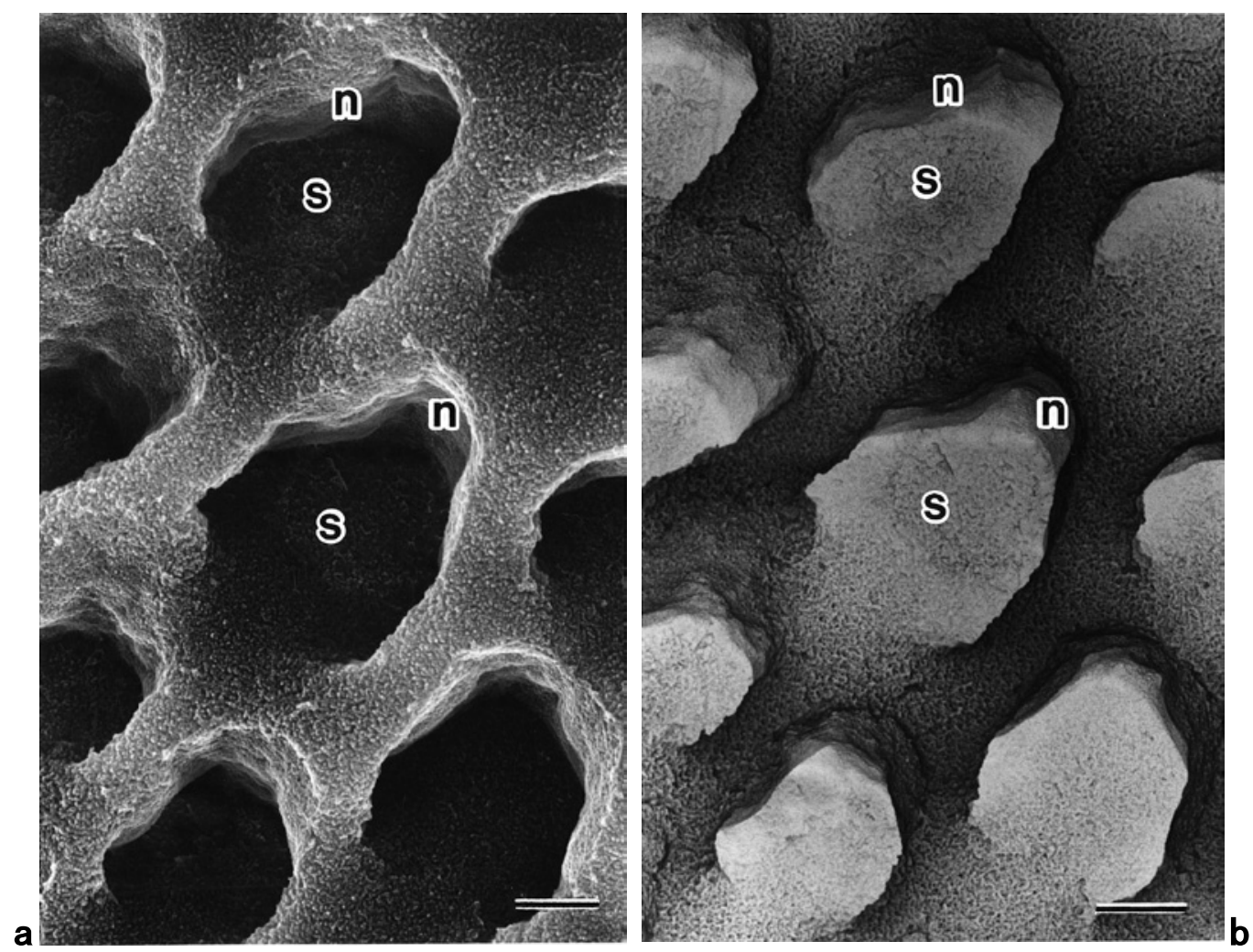

Fig. 5. Scanning electron micrographs of the developing enamel surface after the enamel organ has been removed from the tooth germ using the cell-maceration method. a: The enamel surface consists of a series of pits which have been filled with Tomes' processes. Each pit is composed of a flat (s) and an enclosing face (n). b: Optically reversed photograph of $\mathbf{a}$. Each pit appears to show the contours of the Tomes' processes consisting of secretory faces (s) and round or triangular nonsecretory faces (n).

Scale bars: $1 \mu \mathrm{m}$

prisms were then traced onto the next serial sections. On the section $40 \mu \mathrm{m}$ distant from the base of the prisms, the row of enamel prisms was shown to be arranged in a wavy sine like curve (Fig. 2b). The green and blue prisms became arranged in belt-like zones roughly perpendicular to the occlusal direction, while showing occasional confluences and divergences (Fig. 2b).

On a tangential semithin section from a cervical enamel region (plane TA in Fig.1), the cross-cut Tomes' processes at the initial enamel formation appeared round in profile and arranged in a somewhat irregular pattern (Fig.3a). In the following stages of enamel formation, the direction of the secretory faces of the Tomes' processes could be estimated from the cross-cut profiles and indicated that the secretory faces became twisted to the right or left, and that some rough outlines of the specks of ameloblasts comprised those with similarly inclined Tomes' processes.
In Figure 3b, the Tomes' processes with secretory faces oriented to the right were colored blue, and ones with those oriented to the left were colored green (Fig.3b).

On a tangential semithin section through the ameloblast layer, groups of densely stained cross-sections and lightly stained cross-sections of ameloblasts were discernible as alternating belt-like zones roughly perpendicular to the occlusal direction (Fig.4a). In some areas, the groups of ameloblasts appeared to be arranged in longitudinally oriented sinusoidal rows of a 16-20 ameloblastcycle (Fig.4b).

\section{Scanning electron microscopy}

The exposed surface of the developing enamel showed a series of pits previously filled by Tomes' processes, each consisting of a combination of a flat and an enclosing 

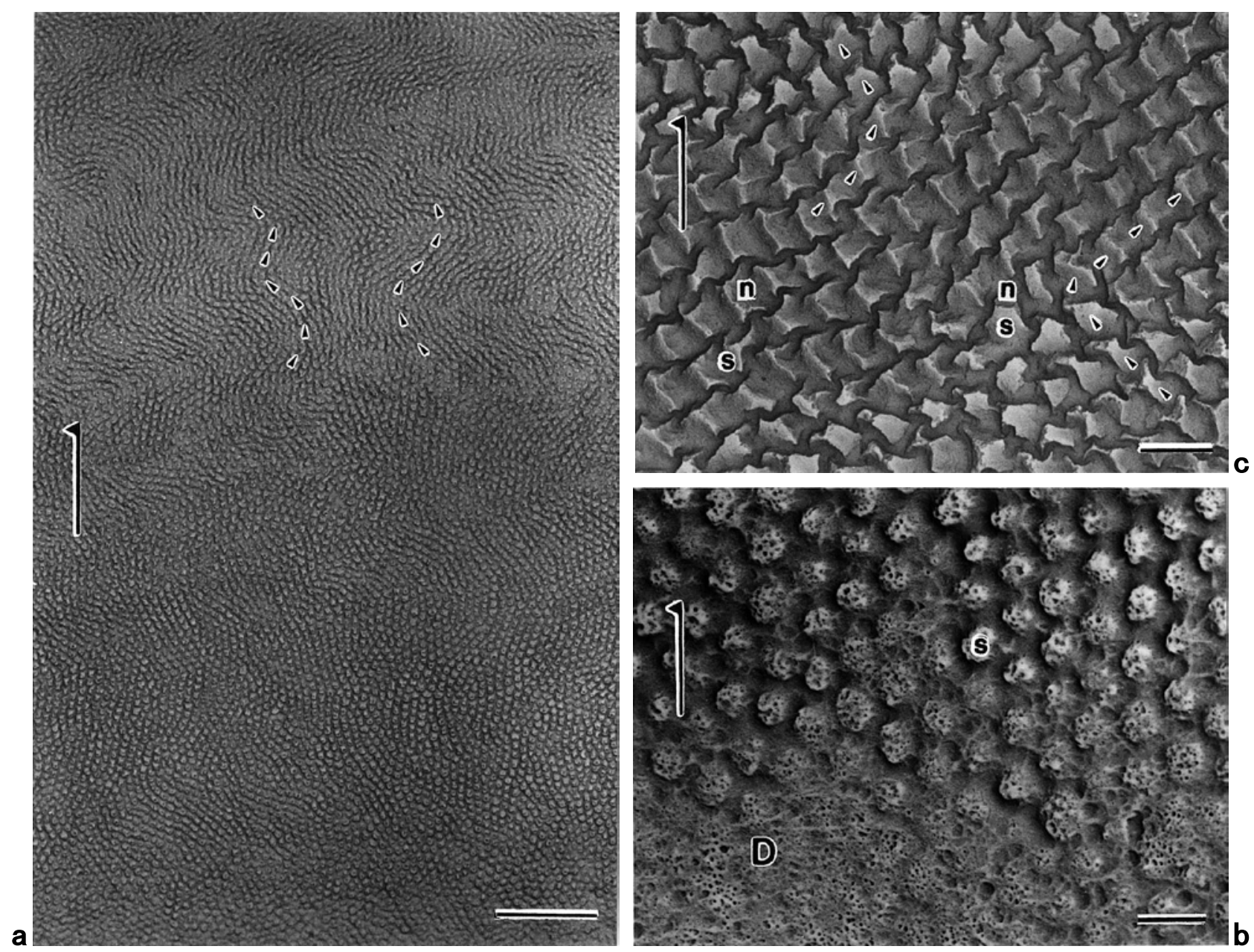

Fig. 6. Reversed photographs of scanning electron micrographs of the developing enamel surface cleaned of cellular components by the cell-maceration method. a: Micrograph showing the exposed surface of the apical end of ameloblasts, where the Tomes' processes are developed. Near the enamel-dentin junction (bottom of figure), Tomes' processes are arranged in a somewhat irregular pattern. Toward the occlusal direction (arrow), Tomes' processes align in rows in the occlusal direction (arrowheads). Scale bar: $50 \mu \mathrm{m}$. b: Enlargement of a. At the initial enamel formation, Tomes' processes are round with round secretory faces (s) and extend perpendicular to the developing enamel surface. D: Dentin. c: Enlargement of a at the more occlusal area of the inner enamel formation where gradual changes in the direction of the secretory faces (s) of the uniformly inclined Tomes' processes are noted (arrowheads). n: nonsecretory face of Tomes' process. s: secretory face of Tomes' process, Scale bars: $7 \mu \mathrm{m}$

face (Fig. 5a). When the white and black image of the photograph was reversed, the flat faces and enclosing faces of pits (Fig. 5a) respectively revealed the contours of the secretory and nonsecretory faces of Tomes' processes (Fig. 5b). A panoramic low magnification scanning electron microscope image (Fig. 6a) showed similar patterns of Tomes' processes as shown in a tangential section parallel to the enamel surface (Fig. 3).
At the initial stage of enamel secretion, Tomes' processes appeared round and arranged in a somewhat irregular pattern with the secretory faces inclined perpendicular to the developing enamel surface (Fig. 6a, b). Toward the occlusal direction, Tomes' processes became aligned in sinusoidal rows along the occlusal direction, with the secretory faces gradually changing their inclination either to the right or to the left (Fig. 6a, c). 


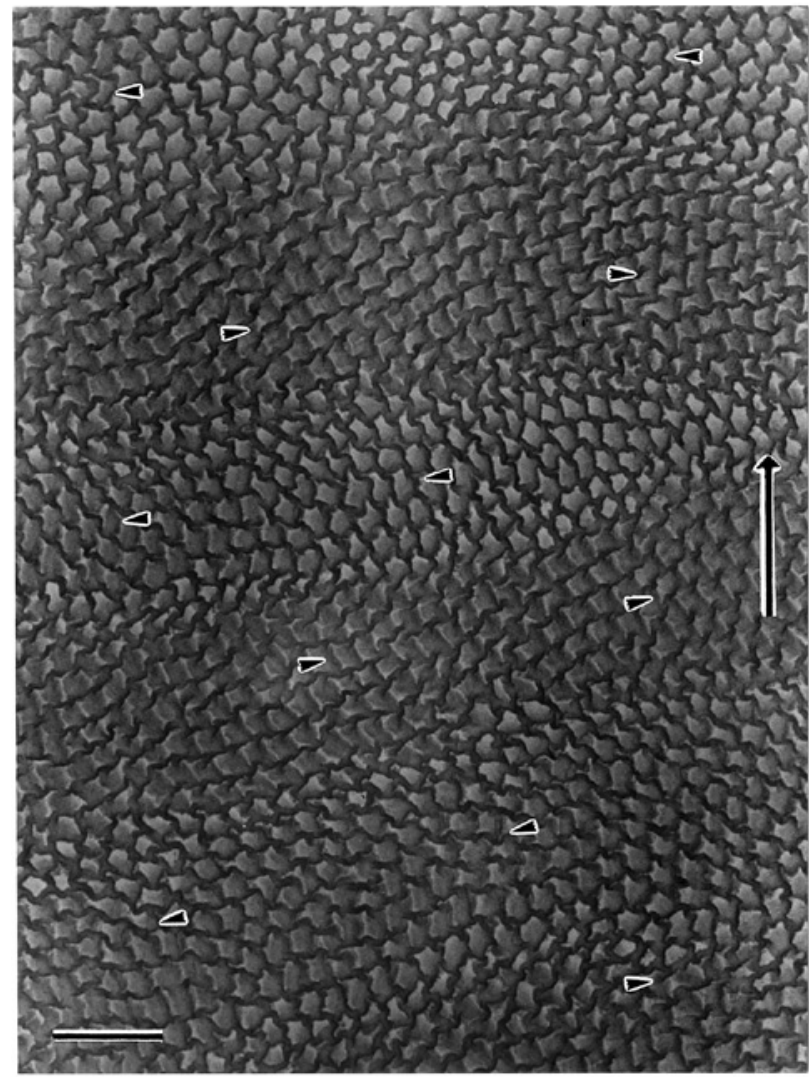

Fig. 7. Reversed photograph of scanning electron micrographs of the exposed surface of developing enamel cleaned of cellular components by the cell-maceration method more occlusally than in Figure 6, $50 \mu \mathrm{m}$ from the enamel-dentin junction. The exposed surface shows belt-like zones running roughly perpendicular to the occlusal direction (arrow). Those belt-like zones consist of Tomes' processes inclined uniformly toward the same sideward direction. Right pointing arrowheads show groups of Tomes' processes oriented to the left. Left pointing arrowheads show groups of Tomes' processes oriented to the right. Scale bar: $20 \mu \mathrm{m}$

Further in the occlusal direction, groups of Tomes' processes inclined uniformly toward the same sideward direction and formed a belt-like arrangement, with neighboring groups of Tomes' processes inclined in the opposite direction (Fig. 7). More occlusally, groups of ameloblasts with similarly inclined secretory faces formed a wavy belt-like arrangement (Fig. 8).

\section{Three-dimensional reconstruction of enamel prisms and groups of prisms}

A row of the numbered enamel prisms and the boundaries of the blue or green groups of enamel prisms, oriented uniformly in one direction as shown in Figure 2, were reconstructed over $5 \mu \mathrm{m}$ (Fig. 9a), $20 \mu \mathrm{m}$ (Fig. 9b), and $45 \mu \mathrm{m}$ (Figs. 9c, d) by the respective data from sections 5-10 $\mu \mathrm{m}$ (Fig. 9a), 5-25 $\mu \mathrm{m}$ (Fig. 9b), and 5-50 $\mu \mathrm{m}$ (Fig. 9c, d) from the enamel-dentin junction, viewed obliquely from the surface. Emerging from the enameldentin junction, a straight row of parallel enamel prisms extended perpendicularly to the enamel-dentin junction. There were some island-like small specks, or groups of enamel prisms oriented in the same direction (Fig. 9a). At $25 \mu \mathrm{m}$ from the enamel-dentin junction, the row of enamel prisms tilted sidewards to the left or right from the enamel-dentin junction (Fig. 9b).

At $50 \mu \mathrm{m}$ from the enamel-dentin junction, the pink colored cut ends of enamel prisms showed a periodic tilted pattern. In one group, the horizontal tilt angles of enamel prisms toward the enamel-dentin junction tended to be larger at the center and smaller near the boundaries (Fig. 9c).

The reconstructed images of blue and green colored groups of enamel prisms displayed as wire-frame outlines allowed an understanding of the expansion of grouping of enamel prisms. Each speck of groups of enamel prisms expanded its boundaries and merged with those of neighboring groups inclined towards the same sideward direction. Consequently, groups of enamel prisms were arranged in numerous belt-like zones perpendicular to the occlusal direction of the tooth (Figs. 9c,d).

\section{Discussion}

One ameloblast forms the body of a single enamel prism, which extends from the enamel-dentin junction to the enamel surface while showing a horizontal sideward shift and longitudinal tilts (Hanaizumi, 1992; Hanaizumi, et al., 1994, 1996, 1998). Thus, the three-dimensional arrangement of the individual enamel prisms represents the overall radial movement of the individual ameloblasts. The present study has shown that the patterns of enamel prism arrangement related to the Hunter-Schreger bands first appear as bundles of enamel prisms oriented in specific sideward directions at $10 \mu \mathrm{m}$ from the enameldentin junction, and that these appear as islands of clusters of cross-cut surfaces of the uniformly oriented 


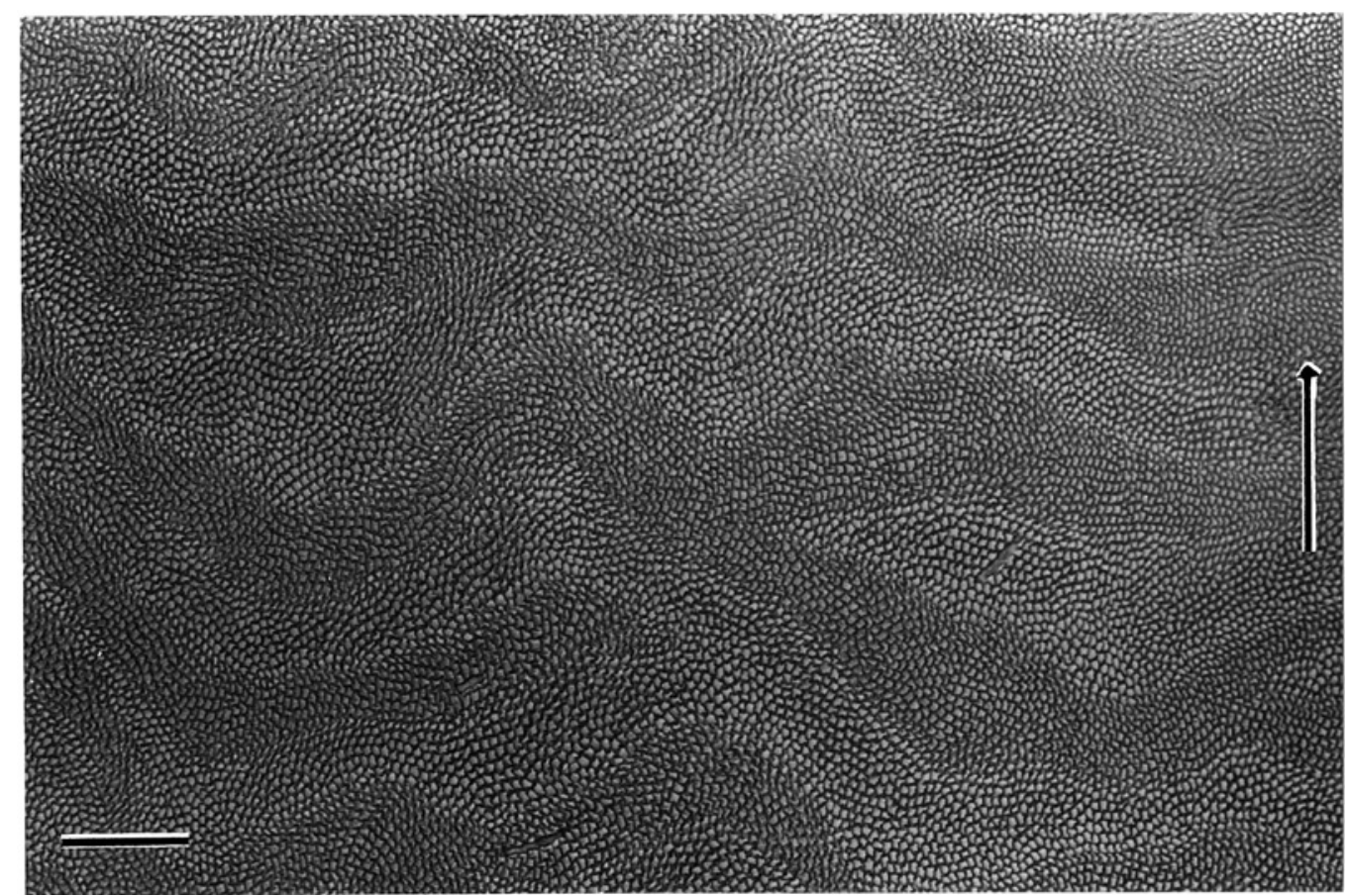

Fig. 8. Reversed photograph of a scanning electron micrograph of the exposed surface of developing enamel cleaned of cellular components by the cell-maceration method. The position is more occlusal than in Figure 7 . The exposed apical surfaces of the ameloblasts consist of wavy belt-like groups with similarly inclined secretory faces of Tomes' processes, nearer to the top of the enamel surface. The occlusal direction is indicated by the arrow. Scale bar: $50 \mu \mathrm{m}$

enamel prisms in tangential sections of the enamel. The reversed SEM images of the exposed surfaces of growing enamel have further indicated that the grouping of secretory ameloblasts with Tomes' processes having similarly oriented secretory surfaces is the key event for the subsequent characteristic arrangement of the enamel prisms related to the Hunter-Schreger bands. In light of current and previous observations of the arrangement of enamel prisms and its relation to Tomes' processes, it is now possible to show a schematic representation of ameloblast groupings and their possible movements in three-dimensions at different levels of the enamel layers.

Up to $10 \mu \mathrm{m}$ away from the enamel-dentin junction, enamel prisms are parallel and perpendicular to the enamel-dentin junction, and show small and round profiles in cross-cut sections. Reversed SEM images of the exposed surfaces of growing enamel corresponding to such area show slightly irregularly arranged round, bubble-like structures of Tomes' processes with the opposing secretory faces perpendicular to the developing enamel surface. This observation suggests that, in the initial process of enamel formation up to $10 \mu \mathrm{m}$ from the enamel-dentin junction, ameloblasts display only a backwards movement without a lateral movement. It is only after the initial $10 \mu \mathrm{m}$ of deepest enamel layer is established that the small groups of ameloblasts start to show Tomes' processes with uniformly inclined secretory faces and hence the characteristic backward movement combined with sideward shifting.

It is obvious by three-dimensional reconstruction of the groups of tilted enamel prisms in current and previous observations that, at the growing enamel surface more than $10 \mu \mathrm{m}$ from the enamel-dentin junction, each speck of groupings of ameloblasts having Tomes' processes with similarly oriented secretory faces expands its boundaries and merges with the neighboring specks of ameloblasts having Tomes' processes with secretory faces inclined roughly in the same sideward direction. Ultimately, these groups of ameloblasts form alternate belt-like zones of those respectively having Tomes' processes with secretory 

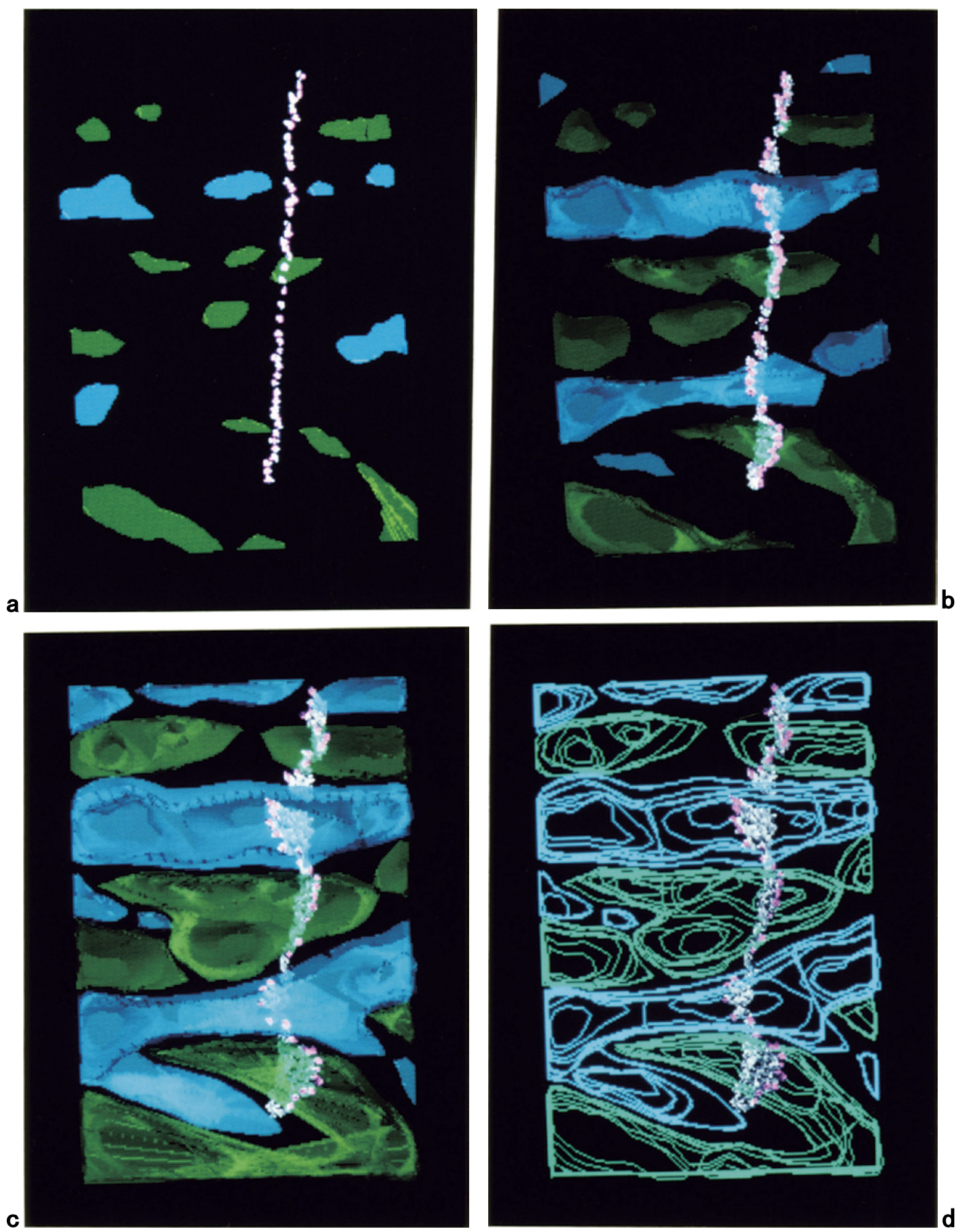

Fig. 9. Legend on the opposite page. 
faces directed to the opposing sideward direction (Fig.11). The present scanning microscopic observations of the exposed apical surfaces of growing enamel have revealed a whole spectrum of developmental and structural changes of Tomes processes extending from the initial to the late stages of enamel matrix formation in a dog tooth germ, providing firm support for the notion of the grouping of ameloblasts based on the surface orientation of Tomes processes and its relation to the HunterSchreger bands.

It is interesting to note that, in accord with the sideward inclination of the secretory faces of Tomes' processes of groups of ameloblasts, the outlines of the cell bodies of respective ameloblasts at the level of the distal terminal web also exhibit a wavy arrangement, creating a sinusoidal curve in the longitudinal direction when viewed in a tangential section. Yokota et al. (2005) conducted immunohistological studies of ameloblasts of dog teeth and reported that, in tangentially sectioned ameloblasts, actin reactions at the distal terminal web appeared as a horizontal belt of honeycomb patterns in which areas without actin reactions were distributed. These authors suggested a correlation between the fluctuating actin reactions in a distal terminal web of ameloblasts with a diverging orientation of enamel prisms and hence the formation of the Hunter-Schreger bands in the enamel layer.

To elucidate the reasons why ameloblasts are grouped

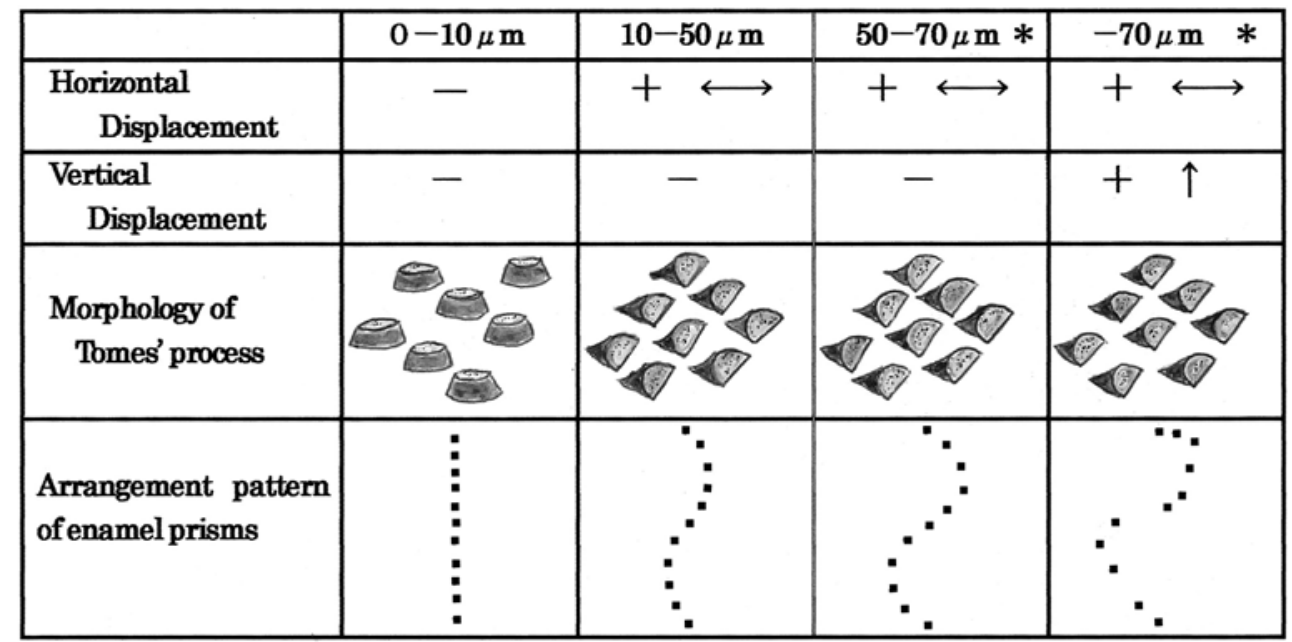

Asterisk, Hanaizumi (1992)

Fig. 10. Summary of the changes in the profiles of Tomes' processes and possible directions of ameloblast sideward displacement based on data from the current and previous studies (Hanaizumi, 1992).

Fig. 9. Reconstruction of the numbered enamel prisms (white area) and the groups of enamel prisms (green and blue areas) in Fig.6, viewed obliquely from the enamel surface. a: 5-10 $\mu \mathrm{m}$ from the enamel-dentin junction. The numbered enamel prisms in the occlusal direction are arranged in parallel, perpendicular to the enamel-dentin junction. Blue and green groups of enamel prisms appear like islands. b: $5-25 \mu \mathrm{m}$ from the enamel-dentin junction. The ends of the enamel prisms (pink area) are tilted sidewards to the left or right. Blue and green groups of prisms show a belt-like arrangement roughly stretching perpendicular to the occlusal direction. c: $5-50 \mu \mathrm{m}$ from the enamel-dentin junction. The pink colored cut ends of enamel prisms show a periodic tilted pattern approaching the enamel surface. In one group, the horizontal tilt angles of prisms toward the enamel-dentin junction are greatest at the center and smaller near the boundaries. d: The same image of rods in $\mathbf{c}$ with the reconstructed images of the blue and green groups of enamel prisms displayed as wire-frames. The specks of grouped enamel prisms expand the boundaries and merge with neighboring groups inclined towards the same sideward direction. 


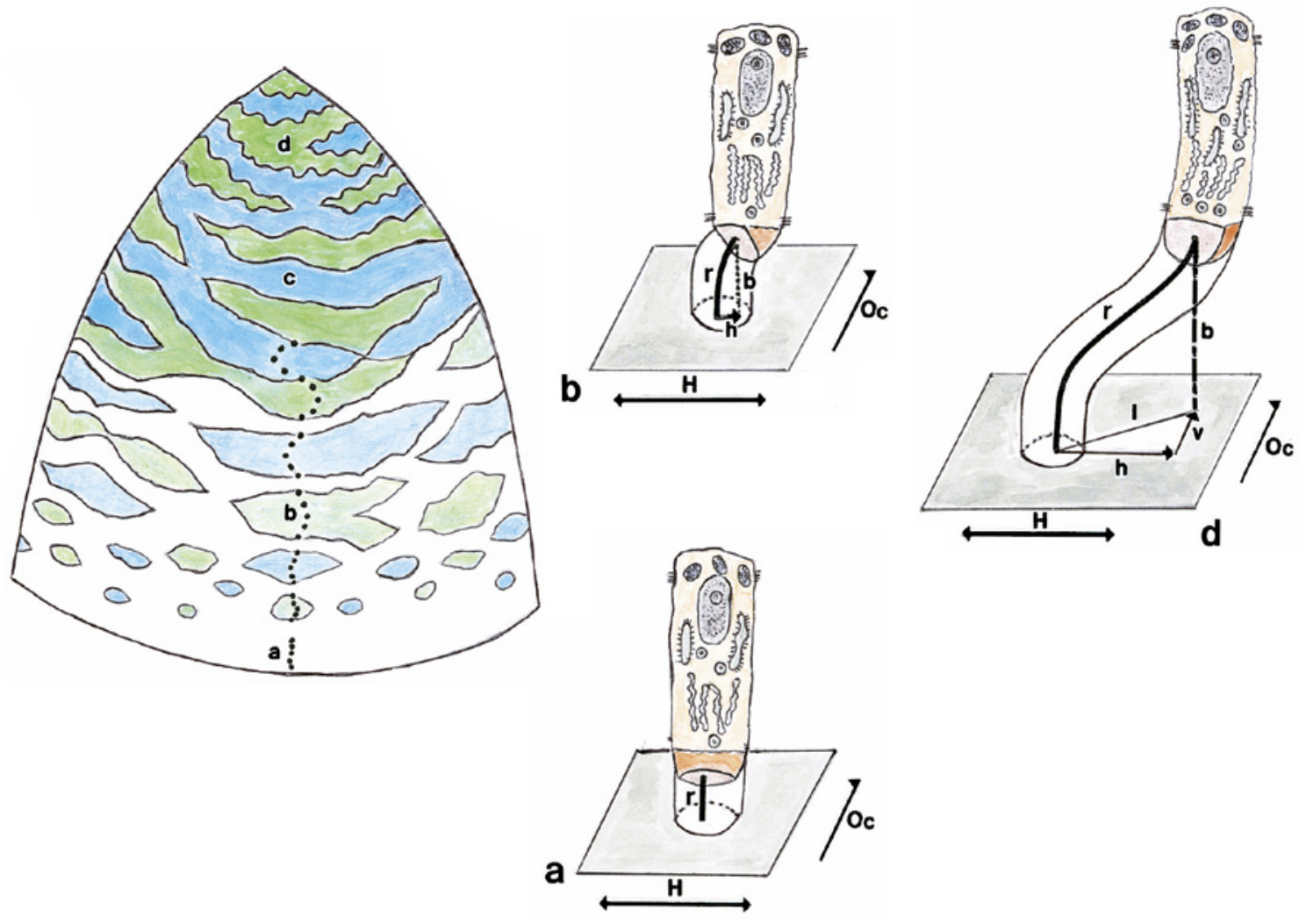

Fig. 11. Schematic representation of the distribution of specks of enamel prisms oriented sideward to the left or right in the early process of enamel formation and correlation of the structures of Tomes processes and spatial movement of ameloblasts at different levels of the enamel layers. The radial movement (r) of ameloblasts from the enamel-dentin junction comprises a backwards movement (b) perpendicular to the enamel-dentin junction and a lateral movement (1) parallel with the enamel-dentin junction. The lateral movement (l) can be divided into horizontal (h) and vertical (v) displacement components parallel to the horizontal $(\mathrm{H})$ or occlusal direction $(\mathrm{Oc})$ of the tooth. Level a: Up to $10 \mu \mathrm{m}$ from the enamel-dentin junction, Tomes' processes are rounded in outline and arranged in a somewhat irregular pattern with the secretory faces perpendicular to the developing enamel surface. The ameloblasts display only a backwards movement and no lateral movement. Level b: At more than $10 \mu \mathrm{m}$ distant from the enamel-dentin junction, the Tomes' process of some groups of ameloblasts start to show a flat secretory face and an enclosing nonsecretory face. The enamel prisms corresponding to these ameloblasts are tilted sidewards to the left or right, and there are small island-like specks of grouped enamel prisms tilting in the same sideward direction. Level c: Each speck of grouped enamel prisms expands and merges with neighboring prisms inclined in the same sideward direction, and groups of enamel prisms become arranged in parallel belt-like zones perpendicular to the occlusal direction of the tooth. Level d: In the enamel more than $70 \mu \mathrm{m}$ apart from the enamel-dentin junction, there is a vertical displacement component in addition to the horizontal displacement, and groups of enamel prisms form a wavy belt-like arrangement (Hanaizumi, 1992). 
to form belt-like arrangements with synchronous inclinations of the secretory faces of Tomes' processes toward the same direction, a more in depth knowledge of the biological events in amelogenesis as well as an understanding of the origins of putative forces causing the sidewards displacement of ameloblasts will be required.

\section{References}

Applebaum E: The arrangement of the enamel rods. New York State Dent J 26: 185-188 (1960).

Boyde A: The structure and development of mammalian enamel. Ph D Thesis, University of London, 1964.

Boyde A: The development of enamel structure. Proc Royal Soc Med 60: 923-928 (1967).

Boyde A: Correction of ameloblast size with enamel prism pattern; Use of scanning electron microscopy to make surface area measurements. Z Zellforsch 93: 583593 (1969).

Erausquin J: The aspect of bands of Schreger in horizontal sections of the enamel. J Dent Res 28: 195-200 (1949).

Gustafson G: The structure of human dental enamel. A histological study by means of incident light, polarized light, phase contrast microscopy, fluorescence microscopy and microhardness tests. Odontol Tidskift 53, Suppl: 1-150 (1945).

Hanaizumi Y: Three-dimensional change in direction and interrelationships among enamel prisms in dog tooth. Arch Histol Cytol 55: 539-550 (1992).

Hanaizumi Y, Shimokobe H, Wakita M: The threedimensional structure of Tomes' processes and their relationship to arrangement of enamel prism in dog teeth. Arch Histol Cytol 57: 129-138 (1994).

Hanaizumi Y, Maeda T, Takano Y: Three-dimensional arrangement of enamel prisms and their relation to the formation of Hunter-Schreger bands in dog tooth. Cell Tissue Res 286: 103-114 (1996).

Hanaizumi Y, Kawano Y, Ohshima H, Hoshino M, Takeuchi K, Maeda T: Three-dimensional direction and interrelationship of prisms in cuspal and cervical enamel of dog tooth. Anat Rec 252: 355-368 (1998).

Heuser H: Die Struktur des menschlichen Zahnschmelzes im oberflächenhistologischen Bild (Replica-Technik). Arch Oral Biol 4: 50-58 (1961).

Hirota F: Prism arrangement in human cusp enamel deduced by X-ray diffraction. Arch Oral Biol 27: 931937 (1982).

Kawai N: The bands of Schreger observed through the enamel surface. Okajimas Fol Anat Jap 26:25-28 (1951).
Kawai N: Comparative anatomy of the bands of Schreger. Okajimas Fol Anat Jap 27: 115-131 (1955).

Kindaichi K, Kagayama M, Akita H, Takahashi N: Developing enamel surface of rhesus monkey and kitten observed by scanning electron microscopy. Jap J Oral Biol 23: $732-738$ (1981).

Koenigswald WV, Rensberger JM, Pretzschner HU: Changes in the tooth enamel of early Paleocene mammals allowing increased diet diversity. Nature 328 : 150-152 (1987).

Kozawa Y: Comparative Histology of Proboscidean molar Enamel. J Stomatol Soc Jap 45: 585-606 (1978).

Kozawa Y: The development and the evolution of mammalian enamel structure. In: Tooth enamel IV (Fearnhead RW, Suga S, ed.) Elsevier, Amsterdam, 1984 (p.432-436)

Lester KS: The bands of Schreger, the role of reflexion. Arch Oral Biol 10: 361-377 (1965).

Mortell JW: Observation of Hunter-Schreger bands. $J$ Dent Res 35: 804-813 (1956).

NIH: Guide for the care use of laboratory animals. US Department of Health and Human Services, 1985.

Nishikawa S: Correlation of the arrangement pattern of enamel rods and secretory ameloblasts in pig and monkey teeth: A possible role of the terminal webs in ameloblasts movement during secretion. Anat Rec 232: 466-478 (1992).

Ohara H, Kindaichi K, Teshima T: The developing enamel surface in rat incisor observed by the resin replica method. Tohoku Univ Dent J 9: 55-59 (1990).

Ohtani O, Ushiki T, Kitamura A: Collagen fibrillar networks as skeletal frameworks: A demonstration by cell-maceration/scanning electron microscope method. Arch Histol Cytol 51: 249-261 (1988).

Osborn JW: The nature of the Hunter-Schreger bands in enamel. Arch Oral Biol 10: 929-993 (1965).

Osborn JW: Evaluation of previous assessments of prism directions in human enamel. J Dent Res 47: 217-223 (1968a).

Osborn JW: Directions and interrelationships of enamel prisms from the sides of human teeth. J Dent Res 47 : 223-232 (1968b).

Osborn JW: Directions and interrelationships of prisms in cuspal and cervical enamel of human teeth. J Dent Res 47: 395-402 (1968c).

Osborn JW: A 3-dimensional model to describe the relation between prism directions, parazones and diazones, and the Hunter-Schreger bands in human tooth enamel. Arch Oral Biol 24: 299-306 (1990).

Skobe Z: The secretory stage of amelogenesis in rat mandibular incisor teeth observed by scanning electron microscopy. Calc Tiss Res 21: 83-103 (1976). 
Skobe Z: Enamel formation in the monkey observed by scanning electron microscopy. Anat Rec 187: 329-334 (1977).

Skobe Z: The pathway of enamel rods at the base of human teeth. J Dent Res 59: 1026-1032 (1980).

Sundstrom B: Schreger bands and their appearance in microradiographs of human dental enamel. Acta Odont Scand 24: 179-194 (1966).

Ushiki T, Ide C: Three-dimensional organization of the collagen fibrils in the rat sciatic nerve as revealed by transmission- and scanning electron microscopy. Cell Tissue Res 260: 175-184 (1990).
Wakita M, Tsuchiya H, Gunji T, Kobayashi S: Threedimensional structure of Tomes' processes and enamel prism formation in the kitten. Arch Histol Jap 44: 285297 (1981).

Warshawsky H, Josephen K, Thylstrup A, Fejerskov O: The development of enamel structure in the rat incisors as compared to the teeth of monkey and man. Anat Rec 200: 371-399 (1981).

Yokota R, Hanaizumi Y, Suzuki K, Chisaka H, Yamamoto H, Sakae T, Hiraga T, Kozawa Y: Immunohistological study on the ameloblasts 'grouping' and 'dancing' forming of enamel structure. Nihon Univ $J$ Oral Sci 31: 77-94 (2005) (in Japanese) 